\title{
A LOWER BOUND FOR THE FIRST PASSAGE TIME DENSITY OF THE SUPRATHRESHOLD ORNSTEIN-UHLENBECK PROCESS
}

\author{
PETER J. THOMAS, ${ }^{*}$ Case Western Reserve University
}

\begin{abstract}
We prove that the first passage time density $\rho(t)$ for an Ornstein-Uhlenbeck process $X(t)$ obeying $\mathrm{d} X=-\beta X \mathrm{~d} t+\sigma \mathrm{d} W$ to reach a fixed threshold $\theta$ from a suprathreshold initial condition $x_{0}>\theta>0$ has a lower bound of the form $\rho(t)>k \exp \left[-p \mathrm{e}^{6 \beta t}\right]$ for positive constants $k$ and $p$ for times $t$ exceeding some positive value $u$. We obtain explicit expressions for $k, p$, and $u$ in terms of $\beta, \sigma, x_{0}$, and $\theta$, and discuss the application of the results to the synchronization of periodically forced stochastic leaky integrate-and-fire model neurons.
\end{abstract}

Keywords: Leaky integrate and fire neuron; Ornstein-Uhlenbeck process; reliability; synchronization; neural model; lower bound

2010 Mathematics Subject Classification: Primary 60J70

Secondary 92C20

\section{Introduction}

The distribution of the first passage time $\tau$ of an Ornstein-Uhlenbeck (OU) process to a fixed boundary is of interest in a variety of fields including mathematical neuroscience, where it describes the interspike interval distribution of a leaky integrate-and-fire model (LIF model) neuron driven by a combination of steady and fluctuating current sources [1]. Unlike the density of the first passage time (FPT) to a fixed boundary for Brownian motion, the FPT density for the OU process is not known in closed form. Explicit expressions are known for the moments of the FPT; in a neuroscience context the mean and variance are respectively related to the rate and variability of neuronal discharge [21], [33]. Conditions guaranteeing the existence and smoothness of the FPT density to constant and moving boundaries have been studied [10], [17].

Intuitively it is perhaps 'obvious' that the FPT density for an OU process is never identically 0, because no matter how removed a time $t$ is from the typical time of first passage, there must exist a set of trajectories of positive measure that make a sufficiently large excursion from the mean behavior that arrival at the boundary is delayed until $t$ or later. It is the purpose of this paper to augment this intuitive argument by providing an explicit positive lower bound valid for the tail of the distribution. We restrict attention to the lower bound problem for the 'suprathreshold' case, i.e. the case in which the initial value $x_{0}$ and the asymptotic mean $x_{\infty}$ of the process are on opposite sides of the threshold value. In the neural context this case corresponds heuristically to that of an LIF model neuron driven by a sufficiently strong injected current that it will reach its firing threshold in finite time, i.e. firing is not dependent on the presence of fluctuations. This case is therefore fundamentally distinct from that of 'stochastic resonance' phenomena, which

Received 26 January 2010; revision received 31 January 2011.

* Postal address: Department of Mathematics, Case Western Reserve University, 10900 Euclid Avenue, Cleveland, OH 44106, USA. Email address: pjthomas@ case.edu 
occur in a subthreshold setting. For the subthreshold case, the initial and asymptotic values of the OU process lie on the same side of the capture boundary. In this case asymptotic results have been proven that characterize the tail of the distribution at large times [6], [14]. In contrast, the author is aware of no results providing strictly positive lower bounds for the FPT density in the suprathreshold case. Such bounds would be of interest in the study of synchronization of neural activity via periodic stimulation, for reasons to be discussed below. Our main results are summarized in Theorem 1.1.

Theorem 1.1. (Positive lower bound theorem.) Let $\{X(t), t \geq 0\}$ be an OU process satisfying the Itô stochastic differential equation

$$
\mathrm{d} X=-\beta X \mathrm{~d} t+\sigma \mathrm{d} W
$$

with initial condition

$$
X(0)=x_{0},
$$

where $\beta, \sigma$, and $x_{0}$ are positive constants, and $W(t)$ is a standard Wiener process. Let $\theta$ be a fixed threshold satisfying $x_{0}>\theta>0$. Then there exist positive constants $k, p$, and $u$ such that the FPT density of the process to the threshold, $\rho_{X}(t)$, satisfies

$$
\rho_{X}(t)>k \exp \left[-p \mathrm{e}^{6 \beta t}\right]
$$

provided that $t>u$.

In particular, for given values of the constants $\beta, \sigma, x_{0}$, and $\theta$, inequality (1.3) is satisfied for the following values of $k, p$, and $u$ :

$$
\begin{aligned}
& k=\frac{1024 \beta}{9 \pi}\left(\frac{x_{0}}{\theta}-1\right), \\
& p=1+\frac{\beta}{32}\left(\frac{\theta}{\sigma}\right)^{2}, \\
& u=\frac{1}{2 \beta} \log \left[1+\left\{8 \vee\left(1+\frac{x_{0}^{2}}{\theta^{2}}\right) \vee\left(\frac{8 \sigma^{2}}{\beta \theta^{2}}\right)\right\}\right] .
\end{aligned}
$$

Here ' $\vee$ ' denotes the maximum operator.

The proof of Theorem 1.1 is obtained from a geometric construction, elaborated in Sections 2 and 3, which exploits the well-known change of variables by which an OU process may be written in terms of a standard Brownian motion [18]. Sato [22] used the same transformation to obtain analytic results on the FPT distribution of the OU process to a constant boundary, in the subthreshold case. While our results use the same change of variables, we cannot obtain the explicit lower bound sought directly from Sato's asymptotic results.

The paper is organized as follows. In Section 2 we introduce the needed change of coordinates and establish a lower bound for the FPT of a standard Brownian motion to a piecewise-linear approximation of a square root boundary. In Section 3 we use these results to establish Theorem 1.1. In Section 4 we discuss potential applications of our result to the synchronization of periodically forced LIF model neurons in the presence of additive noise, as well as related asymptotic results in the literature. 


\section{Bounding the FPT distribution to a square root boundary for Brownian motion}

\subsection{Coordinate transformation}

Let $X(t)$ be an OU process described by (1.1) and (1.2), and let $B(t)$ denote a standard Brownian motion, i.e. $B(t)$ satisfies

$$
\begin{gathered}
\mathrm{d} B=\mathrm{d} W, \\
B(0) \equiv 0,
\end{gathered}
$$

from which $\mathrm{E}[B]=0$ and $\operatorname{var}[B]=t$. We will identify the sample space $\Omega$ for Brownian trajectories with $C[0, \infty)$.

For $t \geq 0$, we have the representation of $X$ in terms of $B$ :

$$
X(t)=x_{0} \mathrm{e}^{-\beta t}+\frac{\sigma \mathrm{e}^{-\beta t}}{\sqrt{2 \beta}} B\left(\mathrm{e}^{2 \beta t}-1\right) .
$$

The problem of finding the FPT distribution for an OU process with a fixed boundary is equivalent to the problem of finding the FPT distribution for a standard Brownian motion to a moving boundary with square root time dependence. Given the parameter $\beta$ for the OU process, (1.1), define an exponentially rescaled time

$$
s=\mathrm{e}^{2 \beta t}-1, \quad t \geq 0 .
$$

Equivalently, for $s \geq 0$, we have $t=(1 / 2 \beta) \log (s+1)$.

Lemma 2.1. Let $X$ and $B$ be as defined in (1.1)-(1.2) and(2.1)-(2.2), with $x_{0}>\theta>0$, and let $s$ be as defined in (2.4). Fix a particular Wiener process trajectory $\omega \in \Omega$. Then $X(t, \omega)>\theta$ for $0 \leq t<\tau$ if and only if

$$
B(s, \omega)>\sqrt{\frac{2 \beta}{\sigma^{2}}}\left(\theta \sqrt{s+1}-x_{0}\right)
$$

for all $0 \leq s<\mathrm{e}^{2 \beta \tau}-1$.

Proof. From (2.3),

$$
\begin{array}{ll} 
& X(t)>\theta \quad \text { for } 0 \leq t<\tau \\
\Longleftrightarrow \quad & x_{0} \mathrm{e}^{-\beta t}+\frac{\sigma \mathrm{e}^{-\beta t}}{\sqrt{2 \beta}} B\left(\mathrm{e}^{2 \beta t}-1\right)>\theta \quad \text { for } 0 \leq t<\tau \\
\Longleftrightarrow \quad & B\left(\mathrm{e}^{2 \beta t}-1\right)>\sqrt{\frac{2 \beta}{\sigma^{2}}}\left(\theta-x_{0} \mathrm{e}^{-\beta t}\right) \mathrm{e}^{\beta t} \quad \text { for } 0 \leq t<\tau \\
\Longleftrightarrow \quad & B(s)>\sqrt{\frac{2 \beta}{\sigma^{2}}}\left(\mathrm{e}^{\beta t} \theta-x_{0}\right) \quad \text { for } 0 \leq s<\mathrm{e}^{2 \beta \tau}-1 \\
& \quad B(s)>\sqrt{\frac{2 \beta}{\sigma^{2}}}\left(\theta \sqrt{s+1}-x_{0}\right) \quad \text { for } 0 \leq s<\mathrm{e}^{2 \beta \tau}-1 .
\end{array}
$$

This concludes the proof.

Note that, when $s=t=0$, we have $B(0) \equiv 0>\left(\sqrt{2 \beta / \sigma^{2}}\right)\left(\theta-x_{0}\right)$, consistent with the assumption that $x_{0}>\theta$. 
Let $t^{\prime}$ be the time of first passage of $X(t)$ to $\theta$. Let $s^{\prime}$ be the time of first passage of $B(s)$ to the boundary

$$
b(s)=\frac{\sqrt{2 \beta}}{\sigma}\left(\theta \sqrt{s+1}-x_{0}\right) .
$$

The following corollary to Lemma 2.1 is immediate.

Corollary 2.1. Let the processes $X$ and $B$ be defined as in Lemma 2.1. Let $b$ be the boundary function given by (2.5), and let s be the rescaled time given by (2.4). Then, as $\mathrm{d} t \rightarrow 0$, the FPT densities of $X$ and $B$ satisfy

$$
\operatorname{Pr}\left(t^{\prime} \in[t, t+\mathrm{d} t)\right)=\operatorname{Pr}\left(s^{\prime} \in[s, s+\mathrm{d} s)\right)
$$

with

$$
\mathrm{d} s=\frac{\mathrm{d} s}{\mathrm{~d} t} \mathrm{~d} t=2 \beta \mathrm{e}^{2 \beta t} \mathrm{~d} t=2 \beta(s+1) \mathrm{d} t .
$$

Consequently $\rho_{X}(t)$, the FPT density for $X$, can be obtained from the FPT density for $B$, $\rho_{B}(s)$, as

$$
\rho_{X}(t)=2 \beta(s+1) \rho_{B}(s)=2 \beta \mathrm{e}^{2 \beta t} \rho_{B}\left(\mathrm{e}^{2 \beta t}-1\right) .
$$

An explicit positive lower bound for $\rho_{B}$ will then provide a positive lower bound for $\rho_{X}$.

\subsection{Piecewise-linear approximation for $b(s)$}

In order to obtain a positive lower bound for $\rho_{B}$, we will need the slope $b^{\prime}(s)$ of the boundary function $b(s)$ :

$$
b^{\prime}(s)=\frac{\sqrt{2 \beta}}{\sigma} \frac{\theta}{2 \sqrt{s+1}}=\frac{\theta}{\sigma} \sqrt{\frac{\beta}{2}} \frac{1}{\sqrt{s+1}} .
$$

In particular, we have $b^{\prime}(0)=\theta(\sqrt{\beta / 2}) / \sigma>0$ and, for all $s \geq 0, b^{\prime}(s)>0$.

Given $s^{\prime}>0$ and a small time $\mathrm{d} s>0$, let $\Omega_{0} \subset \Omega$ be the set of all trajectories in the sample space $\Omega=C[0, \infty)$ with FPT $s^{\prime}$ to the boundary $b(s)$ in the small interval $s^{\prime} \in[s, s+\mathrm{d} s)$. We will decompose the set $\Omega_{0}$ into two subsets and obtain a positive lower bound for the measure of one of them. First we construct a piecewise-linear approximation of the boundary function $b$ (see Figure 1).

Let $L_{1}$ be the half-line $\left\{\left(s, a_{1}+s b_{1}\right) \mid s \geq 0\right\}$, where

$$
\begin{aligned}
& a_{1}=b(0)=\frac{\left(\theta-x_{0}\right) \sqrt{2 \beta}}{\sigma<0}, \\
& b_{1}=b^{\prime}(0)=\frac{(\theta / 2) \sqrt{2 \beta}}{\sigma>0} .
\end{aligned}
$$

The line $L_{1}$ is tangent to the boundary function $b(s)$ at $s=0$. The boundary function has negative second derivative for all $s>0$, so we have $a_{1}+s b_{1}>b(s)$ for all $s>0$. Consequently, every continuous trajectory $B(s)$ that begins at the initial condition $B(0) \equiv 0$ and meets the boundary $b(s)$ at some time $s^{\prime}>0$ must first meet the line $L_{1}$ at some time $s^{\prime \prime}<s^{\prime}$.

Let $s^{\prime}>0$ be given. We define a second boundary line $L_{2}$ as the horizontal line with height

$$
a_{2}=b\left(s^{\prime}\right)=\frac{\sqrt{2 \beta}}{\sigma}\left(\theta \sqrt{s^{\prime}+1}-x_{0}\right) .
$$

Lines $L_{1}$ and $L_{2}$ intersect at the point

$$
s_{*}=\frac{a_{2}-a_{1}}{b_{1}}=2\left(\sqrt{s^{\prime}+1}-1\right) .
$$




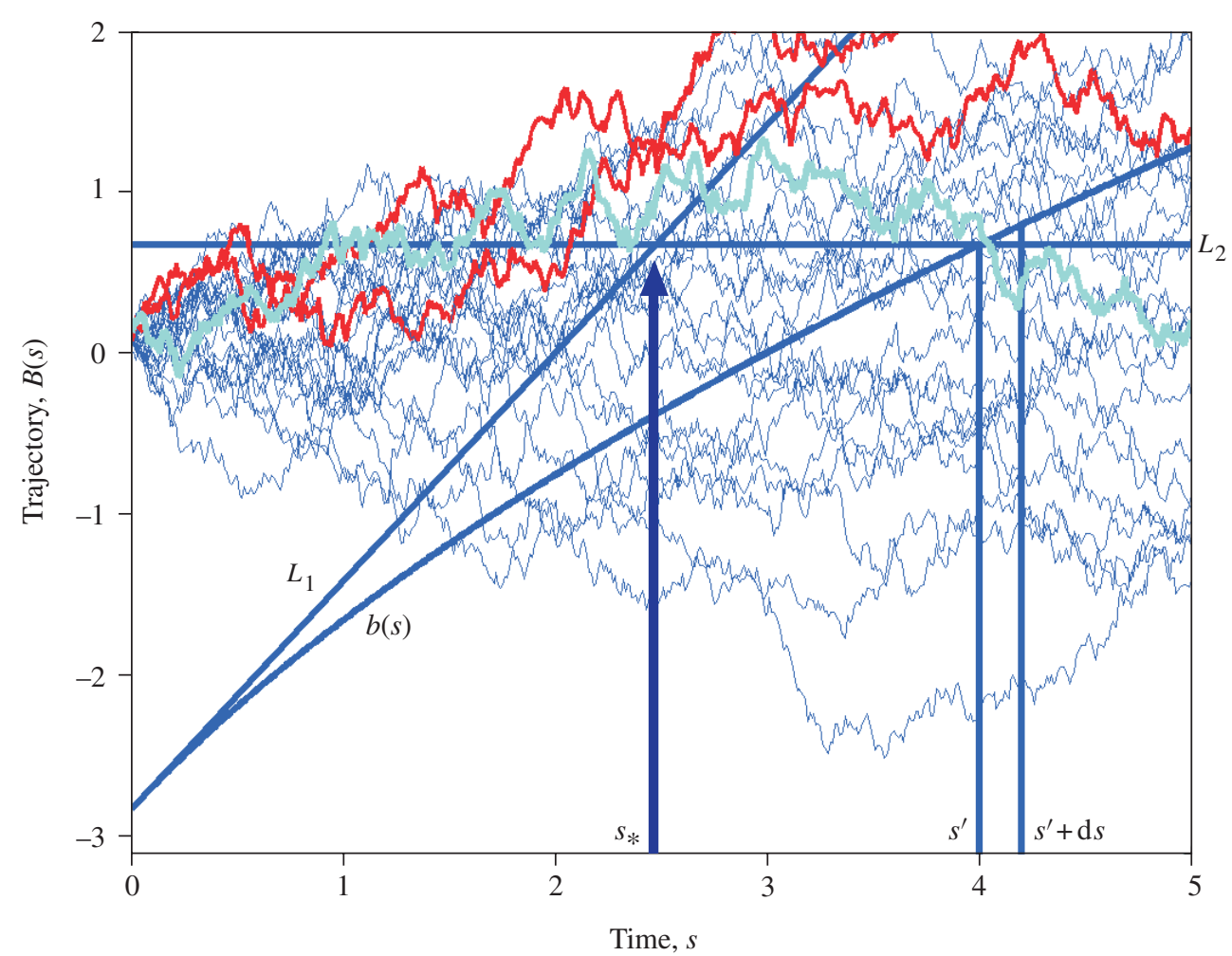

FIGURE 1: Geometric construction of the piecewise-linear boundary approximation, and sample trajectories. Here the boundary $b(s)$ is the arc starting from $b(0) \approx-2.8$, and is given by (2.5) with $\sigma=0.5, \beta=1, x_{0}=2$, and $\theta=1$. The line $L_{1}$ begins at the same location and rises above the arc with slope $b_{1}=\sqrt{2}$. Line $L_{2}$ is horizontal, intersecting line $L_{1}$ at $s_{*}=2 \sqrt{5}-1 \approx 2.47$ and the boundary $b(s)$ at $s^{\prime}=4$. Approximate Brownian trajectories were generated using MATLAB ${ }^{\circledR}$, beginning at $B(0) \equiv 0$ and evolving with noise factor $\sigma=0.5$. Of the twenty-five trajectories shown, three (thick lines) cross $L_{1}$ for the first time at $s^{\prime \prime}>s_{*}$. One of these trajectories (the thick pale (cyan) line) then crosses $L_{2}$ in the interval $\left[s^{\prime}, s^{\prime}+\mathrm{d} s\right)$; here $\mathrm{d} s=0.2$ for illustration. Because $L_{2}$ lies below $b(s)$ for $s>s^{\prime}$, the cyan trajectory must also have its first passage to $b(s)$ within the interval $\left[s^{\prime}, s^{\prime}+\mathrm{d} s\right)$. The cyan trajectory is a typical element of $\Omega_{2}$.

Note that $s_{*}<s^{\prime}$ provided that $s^{\prime}>0$. The duration $\Delta$ of the interval $I=\left[s_{*}, s^{\prime}\right]$ is

$$
\Delta=s^{\prime}-s *=s^{\prime}+2\left(1-\sqrt{s^{\prime}+1}\right)
$$

and satisfies $\Delta+2 \sqrt{\Delta}=s^{\prime}$. Figure 1 illustrates the geometry of $b(s), L_{1}$, and $L_{2}$. We note for future reference that when $s^{\prime}=8, s_{*}=\Delta=4$, and that $s *$ is a strictly increasing function of $s^{\prime}$ for $s^{\prime} \geq 0$.

Given $\mathrm{d} s>0$, we have defined $\Omega_{0}$ to be the collection of all Wiener process trajectories $\omega \in \Omega=C[0, \infty)$ such that the process $X(s, \omega)$ makes its first passage from $X(0) \equiv 0$ to $b(s)$ in the interval $\left[s^{\prime}, s^{\prime}+\mathrm{d} s\right)$. We further define $\Omega_{1}$ to be the subset of $\Omega_{0}$ consisting of those trajectories in $\Omega_{0}$ that make their first passage from $X(0) \equiv 0$ to the line $L_{1}$ at any time $s^{\prime \prime} \geq s_{*}$. Figure 1 shows an example of such a trajectory (the thick pale (cyan) line). Starting from $X(0)=0$, the cyan trajectory remains above the line $L_{1}$ until passing the intersection 
of $L_{1}$ and $L_{2}$ at $s=s_{*}$, after which it crosses $b(s)$ for the first time in the interval $\left[s^{\prime}, s^{\prime}+\mathrm{d} s\right)$. Of the trajectories in $\Omega_{1}$, some-like the trajectory shown in cyan-also cross the line $L_{2}$ somewhere in the interval $\left[s^{\prime}, s^{\prime}+\mathrm{d} s\right.$ ). Denote the set of trajectories in $\Omega_{1}$ with this property by $\Omega_{2}$.

Clearly, $\Omega_{2} \subset \Omega_{1} \subset \Omega_{0}$. Because $b(s)$ is continuous and strictly increasing, every trajectory that remains above line $L_{1}$ until at least $s=s_{*}$ and then intersects line $L_{2}$ for the first time in the interval $\left[s^{\prime}, s^{\prime}+\mathrm{d} s\right)$ must also make its first passage to $b(s)$ in the interval $\left[s^{\prime}, s^{\prime}+\mathrm{d} s\right)$. Hence, if the density of trajectories in $\Omega_{2}$ crossing $L_{2}$ in $\left[s^{\prime}, s^{\prime}+\mathrm{d} s\right)$ is strictly positive, so is the FPT density of all trajectories in $\Omega_{0}$, which is the FPT density of interest. We now derive an explicit positive lower bound on the FPT density of trajectories in $\Omega_{2}$; hence, also a lower bound for the FPT density of trajectories in $\Omega_{0}$.

\subsection{Strictly positive lower bound for $\rho_{B}$}

The FPT density for a standard Brownian motion from $B(0) \equiv 0$ to a boundary moving with constant velocity is well known (see, e.g. [3, p. 221] and [12]). The density of first passage from $B(0) \equiv 0$ to $L_{1}$ at time $s$ is

$$
g_{01}(s)=\frac{\left|a_{1}\right|}{\left(2 \pi s^{3}\right)^{1 / 2}} \exp \left[-\frac{\left(a_{1}+b_{1} s\right)^{2}}{2 s}\right],
$$

where $a_{1}$ and $b_{1}$ are given in (2.6) and (2.7). Similarly, the density of first passage from a starting position $a_{1}+b_{1} s$ at time $s$ to the line $L_{2}$ at time $s^{\prime}>s$ is given by

$$
g_{12}\left(s^{\prime} \mid s\right)=\frac{\left|\left(a_{1}+b_{1} s\right)-a_{2}\right|}{\left(2 \pi\left(s^{\prime}-s\right)^{3}\right)^{1 / 2}} \exp \left[-\frac{\left(\left(a_{1}+b_{1} s\right)-a_{2}\right)^{2}}{2\left(s^{\prime}-s\right)}\right] .
$$

Because of the almost-sure continuity of Brownian trajectories, we may write the FPT density $g_{2}\left(s^{\prime}\right)$ for trajectories in $\Omega_{2}$ from initial position $B(0) \equiv 0$ to line $L_{2}$ at time $s^{\prime}$ as a convolution of $g_{01}$ with $g_{12}$. Using $l_{1}(s)$ to denote $\left(a_{1}+b_{1} s\right)$, we write

$$
\begin{aligned}
g_{2}\left(s^{\prime}\right) & =\int_{s=s_{*}}^{s^{\prime}} g_{01}(s) g_{12}\left(s^{\prime} \mid s\right) \mathrm{d} s \\
& =\frac{\left|a_{1}\right|}{2 \pi} \int_{s_{*}}^{s^{\prime}} \frac{l_{1}(s)-a_{2}}{\left(\left(s^{\prime}-s\right) s\right)^{3 / 2}} \exp \left[-\frac{l_{1}(s)^{2}}{2 s}-\frac{\left(l_{1}(s)-a_{2}\right)^{2}}{2\left(s^{\prime}-s\right)}\right] \mathrm{d} s,
\end{aligned}
$$

where the upper endpoint $\left(s^{\prime}\right)$ and lower endpoint $\left(s_{*}\right)$ for the integration both depend on $s^{\prime}$, as does the height of $L_{2}$, namely $a_{2}=b\left(s^{\prime}\right)$. The integrand in (2.10) approaches 0 at both endpoints, but is strictly positive in the interior of the interval $I=\left\{s \mid s_{*} \leq s \leq s^{\prime}\right\}$. To obtain a positive lower bound for the integral, we rewrite the integrand as a product of three factors:

$$
\begin{aligned}
g_{2}\left(s^{\prime}\right) & =\frac{\left|a_{1}\right|}{2 \pi} \int_{s \in I} F_{1}\left(s, s^{\prime}\right) F_{2}\left(s, s^{\prime}\right) F_{3}\left(s, s^{\prime}\right) \mathrm{d} s, \\
F_{1}\left(s, s^{\prime}\right) & =a_{1}-a_{2}\left(s^{\prime}\right)+b_{1} s, \\
F_{2}\left(s, s^{\prime}\right) & =\left(s\left(s^{\prime}-s\right)\right)^{-3 / 2}, \\
F_{3}\left(s, s^{\prime}\right) & =\exp \left[-\frac{\left(s^{\prime}-s\right) l_{1}^{2}(s)+s\left(l_{1}(s)-a_{2}\right)^{2}}{2 s\left(s^{\prime}-s\right)}\right]=\exp \left[\frac{-\beta}{4 \sigma^{2}} \frac{Q_{1}\left(s, s^{\prime}\right)}{Q_{2}\left(s, s^{\prime}\right)}\right],
\end{aligned}
$$


where we introduce the notation

$$
\begin{aligned}
Q_{1}\left(s, s^{\prime}\right)= & \left(s^{\prime} l_{1}^{2}(s)-2 s l_{1}(s) a_{2}+s a_{2}^{2}\right) \frac{2 \sigma^{2}}{\beta} \\
= & s^{2} \theta\left(4 x_{0}+\theta\left(s^{\prime}-4 \sqrt{1+s^{\prime}}\right)\right)+4 s^{\prime}\left(x_{0}-\theta\right)^{2} \\
& -4 s\left(x_{0}^{2}+\left(s^{\prime}-2\right) x_{0} \theta+\left(-1-2 s^{\prime}+2 \sqrt{1+s^{\prime}}\right) \theta^{2}\right), \\
Q_{2}\left(s, s^{\prime}\right)= & s\left(s^{\prime}-s\right) .
\end{aligned}
$$

By construction, $F_{1}\left(s_{*}, s^{\prime}\right) \equiv 0$ for all $s^{\prime}>0$ (recalling that $s_{*}$ depends on $\left.s^{\prime}\right)$ and $F_{1}\left(s, s^{\prime}\right)>0$ for $s>s_{*}$. On the other hand, $F_{3}\left(s, s^{\prime}\right) \rightarrow 0$ as $s \rightarrow s^{\prime}$. Thus, the integrand approaches 0 at both ends of the interval of integration. In order to obtain a strictly positive lower bound on the integral, we restrict integration to a subinterval of $\left[s_{*}, s^{\prime}\right]$. Let $\eta$ and $v$ be chosen in the interval $0<\eta<v<1$, and set

$$
s_{1}=s_{*}+\eta \Delta, \quad s_{2}=s_{*}+v \Delta,
$$

where, as above, $\Delta=\left(s^{\prime}-s_{*}\right)$ is the duration of the integration interval. Then $s_{*}<s_{1}<$ $s_{2}<s^{\prime}$. Let $I^{\prime}$ denote the interval $I^{\prime}=\left[s_{1}, s_{2}\right] \subset I$. Each factor $F_{1}, F_{2}$, and $F_{3}$ is nonnegative on the interval $I$. Consequently, we have the inequality

$$
g_{2}\left(s^{\prime}\right) \geq \frac{\left|a_{1}\right|}{2 \pi} \int_{s \in I^{\prime}} F_{1}\left(s, s^{\prime}\right) F_{2}\left(s, s^{\prime}\right) F_{3}\left(s, s^{\prime}\right) \mathrm{d} s .
$$

We consider each factor in turn.

The factor $F_{1}\left(s, s^{\prime}\right)$ is strictly increasing in $s$ for all $s, s^{\prime}$, so, for all $s \in I^{\prime}$, we have

$$
F_{1}\left(s, s^{\prime}\right) \geq F_{1}\left(s_{1}, s^{\prime}\right)=\eta \frac{\theta \sqrt{2 \beta}}{2 \sigma} \Delta .
$$

The quadratic $Q_{2}\left(s, s^{\prime}\right)$, which also appears in the factor $F_{2}\left(s, s^{\prime}\right)=Q_{2}\left(s, s^{\prime}\right)^{-3 / 2}$, has a global maximum (in $s$ ) of $\left(s^{\prime}\right)^{2} / 4$ at $s=s^{\prime} / 2$. In the range $s \in\left[s_{1}, s_{2}\right], Q_{2}\left(s, s^{\prime}\right)$ is strictly positive. Consequently for all $s \in I^{\prime}$, we have

$$
F_{2}\left(s, s^{\prime}\right) \geq F_{2}\left(\frac{s^{\prime}}{2}, s^{\prime}\right)=\frac{8}{\left(s^{\prime}\right)^{3}} .
$$

From (2.10), it is clear that both $Q_{1}\left(s, s^{\prime}\right)$ and $Q_{2}\left(s, s^{\prime}\right)$ are strictly positive for $s \in I^{\prime}$. The factors $\beta$ and $\sigma$ are also positive; therefore, for $s \in I^{\prime}$,

$$
F_{3}\left(s, s^{\prime}\right) \geq \exp \left[-\frac{\beta}{4 \sigma^{2}} \frac{\max _{I^{\prime}} Q_{1}\left(s, s^{\prime}\right)}{\min _{I^{\prime}} Q_{2}\left(s, s^{\prime}\right)}\right] .
$$

As established in the next lemma, $Q_{1}$ is just a quadratic function of $s$ with second derivative that is guaranteed to be positive, provided that $s^{\prime}>8$.

Lemma 2.2. If $s^{\prime}>8$ and $x_{0}>\theta>0$, then the quadratic function $Q_{1}\left(s, s^{\prime}\right)$ defined in (2.12) has positive second derivative with respect to $s$.

Proof. The second derivative of $Q_{1}$ with respect to $s$ is constant in $s, \partial^{2} Q_{1} / \partial s^{2}=2 \theta\left(4 x_{0}+\right.$ $\left.\theta\left(s^{\prime}-4 \sqrt{1+s^{\prime}}\right)\right)$. Suppose that $s^{\prime}>8$. Then $\left(s^{\prime}\right)^{2}>8 s^{\prime}$, so $\left(4+s^{\prime}\right)^{2}>16+16 s^{\prime}$. Taking square roots, we obtain $4+s^{\prime}>4 \sqrt{1+s^{\prime}}$, which means that $1>\sqrt{1+s^{\prime}}-s^{\prime} / 4$ and, since $\theta>0$ by assumption, $\theta>\theta\left(\sqrt{1+s^{\prime}}-s^{\prime} / 4\right)$. By the assumption on $x_{0}, x_{0}>\theta>$ $\theta\left(\sqrt{1+s^{\prime}}-s^{\prime} / 4\right)$. Consequently, $x_{0}+\theta\left(s^{\prime} / 4-\sqrt{1+s^{\prime}}\right)>0$, from which the conclusion follows. 
Because $Q_{1}\left(s, s^{\prime}\right)$ has constant positive second derivative with respect to $s$, it achieves its maximum on $I^{\prime}$ either at $s_{1}$ or at $s_{2}$. The quadratic $Q_{2}\left(s, s^{\prime}\right)$ has constant negative second derivative with respect to $s$ and achieves its minimum at one of the endpoints as well. Therefore, we may conclude that

$$
F_{3}(s) \geq \exp \left[-\frac{\beta}{4 \sigma^{2}}\left(\frac{Q_{1}\left(s_{1}\right) \vee Q_{1}\left(s_{2}\right)}{Q_{2}\left(s_{1}\right) \wedge Q_{2}\left(s_{2}\right)}\right)\right],
$$

where ' $\vee$ ' denotes the greater and ' $\wedge$ ' denotes the lesser of the two arguments. For compactness, here and henceforth, we write $Q_{i}(s)$ for $Q_{i}\left(s, s^{\prime}\right)$, leaving the dependence on $s^{\prime}$ implicit.

In terms of the quadratics $Q_{1}$ and $Q_{2}$, and the given constants, we therefore have the following lower bound for the FPT density at time $s^{\prime}$ :

$$
g_{2}\left(s^{\prime}\right) \geq \frac{\left|a_{1}\right|}{2 \pi}(v-\eta) \Delta\left(\eta \frac{\theta \sqrt{2 \beta}}{2 \sigma} \Delta\right) \frac{8}{\left(s^{\prime}\right)^{3}} \exp \left[-\frac{\beta}{4 \sigma^{2}}\left(\frac{Q_{1}\left(s_{1}\right) \vee Q_{1}\left(s_{2}\right)}{Q_{2}\left(s_{1}\right) \wedge Q_{2}\left(s_{2}\right)}\right)\right],
$$

provided that $s^{\prime}>8$.

Note that, for $s^{\prime}>8, \Delta\left(s^{\prime}\right)>\frac{2}{3}\left(s^{\prime}-2\right)>4$. Since $\left|a_{1}\right|=\left(x_{0}-\theta\right) \sqrt{2 \beta} / \sigma>0$, we may write

$$
\begin{aligned}
g_{2}\left(s^{\prime}\right) & >(v-\eta) \frac{\left(x_{0}-\theta\right) \sqrt{2 \beta}}{2 \pi \sigma}\left(\frac{8}{9} \frac{\theta \sqrt{2 \beta}}{\sigma} \eta\right) \frac{8}{\left(s^{\prime}\right)^{3}} \exp \left[-\frac{\beta}{4 \sigma^{2}}\left(\frac{Q_{1}\left(s_{1}\right) \vee Q_{1}\left(s_{2}\right)}{Q_{2}\left(s_{1}\right) \wedge Q_{2}\left(s_{2}\right)}\right)\right] \\
& =(v-\eta) \eta \frac{64 \beta \theta}{9 \pi \sigma^{2}} \frac{x_{0}-\theta}{\left(s^{\prime}\right)^{3}} \exp \left[-\frac{\beta}{4 \sigma^{2}}\left(\frac{Q_{1}\left(s_{1}\right) \vee Q_{1}\left(s_{2}\right)}{Q_{2}\left(s_{1}\right) \wedge Q_{2}\left(s_{2}\right)}\right)\right] .
\end{aligned}
$$

For any number $\alpha, 0<\eta \leq \alpha \leq v<1$, we may write a point $s \in I^{\prime}$ as $s=s_{*}+\alpha\left(s^{\prime}-s_{*}\right)$ and $\left(s^{\prime}-s\right)=(1-\alpha)\left(s^{\prime}-s_{*}\right)$. Therefore, we can write $Q_{2}(s)$ as $Q_{2}(s)=(1-\eta) s_{*}\left(s^{\prime}-\right.$ $\left.s_{*}\right)+\eta(1-\eta)\left(s^{\prime}-s_{*}\right)^{2}$. When $s^{\prime}=8$, we have $\Delta=4$ and $s_{*}=4$ from (2.8) and (2.9). Both $\Delta$ and $s_{*}$ are strictly increasing as functions of $s^{\prime}$, so, for $s^{\prime}>8$, we have $\Delta>4$ and $s_{*}>4$. Therefore, for $s^{\prime}>8$, we have

$$
Q_{2}\left(s_{1}\right) \wedge Q_{2}\left(s_{2}\right)=16\left(1-v^{2}\right) .
$$

It remains to obtain an estimate on the quadratic $Q_{1}$.

Lemma 2.3. Assume that $x_{0}>\theta>0$. Fix the constants $0<v<\eta<1, s_{1}=s_{*}+\eta \Delta$, and $s_{2}=s_{*}+v \Delta$, where $\Delta=\left(s^{\prime}-s_{*}\right)$ and $s_{*}$ is as in (2.8). Let $Q_{1}$ be given by (2.12), and let $s^{\prime}>8 \vee\left(1+x_{0}^{2} / \theta^{2}\right)$. Then

$$
Q_{1}\left(s_{1}\right) \vee Q_{1}\left(s_{2}\right) \leq \Delta^{2} \theta^{2} s^{\prime} .
$$

Proof. For any number $0 \leq \alpha \leq 1$, we have a corresponding point $s \in I$ for which $s=s_{*}+\alpha\left(s^{\prime}-s_{*}\right)$ and $\left(s^{\prime}-s\right)=(1-\alpha)\left(s^{\prime}-s_{*}\right)$. As shown in Lemma 2.2, $Q_{1}$ has constant positive second derivative with respect to $s$ when $s^{\prime}>8$. Hence, its largest value for $\alpha \in[0,1]$ exceeds its largest value for $\alpha \in[\nu, \eta]$. From (2.12) we obtain

$$
\frac{Q_{1}(s) \beta}{2 \sigma^{2}}=\left(s^{\prime}-s\right) l_{1}^{2}(s)+s\left(l_{1}(s)-a_{2}\right)=s^{\prime} l_{1}^{2}-s l_{1}^{2}+s l_{1}^{2}-2 s a_{2} l_{1}+s a_{2}^{2} .
$$

Note that, by construction, $l_{1}(s)=b_{1}\left(s-s_{*}\right)+a_{2}$. Therefore,

$$
\begin{aligned}
\frac{Q_{1}(s) \beta}{2 \sigma^{2}} & =\left(s^{\prime}-s\right)\left(b_{1}\left(s-s_{*}\right)+a_{2}\right)^{2}+s\left(b_{1}\left(s-s_{*}\right)\right)^{2} \\
& =\left(s^{\prime}-s\right)\left(b_{1}^{2}\left(s-s_{*}\right)^{2}+2 b_{1} a_{2}\left(s-s_{*}\right)+a_{2}^{2}\right)+s b_{1}^{2}\left(s-s_{*}\right)^{2} .
\end{aligned}
$$


Write $s^{\prime}-s=(1-\alpha) \Delta$ and $s-s_{*}=\alpha \Delta$. Then

$$
\begin{aligned}
\frac{Q_{1}(s(\alpha)) \beta}{2 \sigma^{2}}= & (1-\alpha) \Delta\left(b_{1}^{2} \alpha^{2} \Delta^{2}+2 b_{1} a_{2} \alpha \Delta+a_{2}^{2}\right)+\left(s^{\prime}-(1-\alpha) \Delta\right)\left(\alpha^{2} \Delta^{2}\right) b_{1}^{2} \\
= & \Delta\left(2(1-\alpha) \alpha b_{1} a_{2} \Delta+(1-\alpha) a_{2}^{2}+s^{\prime} b_{1}^{2} \alpha^{2} \Delta\right) \\
= & \Delta \frac{2 \beta}{\sigma^{2}}\left((1-\alpha) \alpha \theta\left(\theta \sqrt{s^{\prime}+1}-x_{0}\right)+(1-\alpha)\left(\theta \sqrt{s^{\prime}+1}-x_{0}\right)^{2}\right. \\
& \left.+\frac{\alpha^{2} \Delta s^{\prime} \theta^{2}}{4}\right) \\
Q_{1}\left(s_{*}\right)= & Q_{1}\left(\left.s(\alpha)\right|_{\alpha=0}\right)=4 \Delta\left(\theta \sqrt{s^{\prime}+1}-x_{0}\right)^{2} \\
Q_{1}\left(s^{\prime}\right)= & Q_{1}\left(\left.s(\alpha)\right|_{\alpha=1}\right)=\Delta^{2} \theta^{2} s^{\prime} .
\end{aligned}
$$

For $s^{\prime}>8$, we have $\Delta>4$, as shown previously. Therefore, for $s^{\prime}>8$, we have the inequalities

$$
\begin{aligned}
\sqrt{\frac{s^{\prime}}{s^{\prime}+1}}<1<\frac{\sqrt{\Delta}}{2}, \quad 0>\frac{1}{2 \sqrt{s^{\prime}+1}}-\frac{\sqrt{\Delta}}{4 \sqrt{s^{\prime}}}, \\
1>3-2 \sqrt{2}>\sqrt{s^{\prime}+1}-\frac{1}{2} \sqrt{\Delta s^{\prime}},
\end{aligned}
$$

since $\sqrt{s^{\prime}+1}-\frac{1}{2} \sqrt{\Delta s^{\prime}}=3-2 \sqrt{2}$ when $s^{\prime}=8$ and

$$
\frac{\mathrm{d}}{\mathrm{d} s}\left(\sqrt{s+1}-\frac{1}{2} \sqrt{\Delta s}\right)=\frac{1}{2 \sqrt{s+1}}-\frac{\sqrt{\Delta}}{4 \sqrt{s}} .
$$

Therefore,

$$
\begin{gathered}
x_{0}>\theta>\theta \sqrt{s^{\prime}+1}-\frac{\theta}{2} \sqrt{\Delta s^{\prime}}, \\
\frac{1}{2} \sqrt{\Delta s^{\prime}} \theta>\theta \sqrt{s^{\prime}+1}-x_{0}, \\
\frac{1}{4} \Delta s^{\prime} \theta^{2}>\left(\theta \sqrt{s^{\prime}+1}-x_{0}\right)^{2}, \\
Q_{1}\left(s^{\prime}\right)>Q_{1}\left(s_{*}\right) .
\end{gathered}
$$

Inequality (2.14) follows from (2.13) because $\left(\theta \sqrt{s^{\prime}+1}-x_{0}\right)>0$, by the assumption that $s^{\prime}>\left(1+x_{0}^{2} / \theta^{2}\right)$. Therefore,

$$
Q_{1}\left(s_{1}\right) \vee Q_{1}\left(s_{2}\right) \leq Q_{1}\left(s^{\prime}\right) \leq \Delta^{2} \theta^{2} s^{\prime},
$$

as was to be shown.

We are now able to state Lemma 2.4 which leads directly to Theorem 1.1.

Lemma 2.4. Let $x_{0}>\theta>0$, and let $s^{\prime}$ be sufficiently large that

$$
s^{\prime}>8 \vee\left(1+\frac{x_{0}^{2}}{\theta^{2}}\right) \vee \frac{8 \sigma^{2}}{\beta \theta^{2}} .
$$

Then, for the standard Brownian motion given by (2.1) and (2.2), the conditional FPT density $g_{2}$ defined by (2.11) for $B(s)$ to arrive at the boundary $b(s)$ given by (2.5) satisfies

$$
g_{2}\left(s^{\prime}\right)>\frac{512}{9 \pi}\left(\frac{x_{0}}{\theta}-1\right)\left(s^{\prime}\right)^{-6} \exp \left[-\frac{\beta}{32}\left(\frac{\theta}{\sigma}\right)^{2}\left(s^{\prime}\right)^{3}\right] .
$$


Proof. Lemmas 2.2 and 2.3 and the subsequent remarks establish that, when $s^{\prime}>8 \vee(1+$ $\left.x_{0}^{2} / \theta^{2}\right)$, the density $g_{2}$ defined by (2.11) satisfies

$$
g_{2}\left(s^{\prime}\right) \geq A \eta(v-\eta) \exp \left[-\frac{2 B}{1-v^{2}}\right],
$$

where the parameters $\eta$ and $v$ may be chosen arbitrarily subject to the constraints $0<\eta<v<1$, and the positive terms $A$ and $B$ (which depend on $s^{\prime}$ ) are given by

$$
A\left(s^{\prime}\right)=\frac{64 \beta \theta\left(x_{0}-\theta\right)}{9 \pi \sigma^{2}\left(s^{\prime}\right)^{3}}, \quad B\left(s^{\prime}\right)=\frac{\beta \theta^{2} \Delta^{2} s^{\prime}}{128 \sigma^{2}} .
$$

Let us rewrite the right-hand side of (2.15) as $g_{2}=A M(\eta, v)$, introducing

$$
M(\eta, v)=\eta(v-\eta) \exp \left[-\frac{2 B}{1-v^{2}}\right] .
$$

Because the choices of $\eta$ and $v$ are arbitrary, within the constraints, $g_{2}$ must be bounded below by the supremum of $M$ over the region $0<\eta<v<1$. Clearly, $M$ is positive and differentiable within this region, and $M \rightarrow 0$ as $(\eta, v)$ approach any of the boundaries $\eta=0, v=1, \eta=v$. So the supremum will occur at a point $\left(\eta_{+}, \nu_{+}\right)$in the interior of the constraint region. Setting $\partial M / \partial \eta$ to 0 to find the critical point gives $\nu_{+}=2 \eta_{+}$. Substituting this back into (2.16) and differentiating gives $\nu_{+}=\sqrt{B+1-\sqrt{B^{2}+B}}$. Substituting again to find the maximum value yields

$$
M_{\max }=\frac{1-C}{4} \exp \left[-\frac{2 B}{C}\right]
$$

where we introduce

$$
C=\sqrt{B^{2}+2 B}-B .
$$

Note that, for all $B>0$, we have $0<C<1$. The value of $C$ increases monotonically and $C \rightarrow 1$ from below as $B \rightarrow \infty$. Since $B$ is directly proportional to $s^{\prime}$, we can find a value of $s^{\prime}$ sufficiently large that $C$ exceeds any value less than 1 . For $B>\frac{1}{4}$, for example, we have $C>\frac{1}{2}$. Consequently, we can bound below the exponential factor in (2.17):

$$
\exp \left[-\frac{2 B}{C}\right] \geq \exp [-4 B]
$$

whenever $B>\frac{1}{4}$.

The factor $(1-C) \rightarrow 0$ as $B \rightarrow \infty$. But provided $B \geq 1$ we have the following:

$$
\begin{gathered}
8 B^{2}-8 B+1>0, \\
\left(4 B^{2}+4 B-1\right)^{2}>16 B^{2}\left(B^{2}+2 B\right), \\
4 B^{2}+4 B-1>4 B \sqrt{B^{2}+2 B}, \\
1-C=1+B-\sqrt{B^{2}+2 B}>\frac{1}{4 B} .
\end{gathered}
$$

Inequality (2.20) follows from (2.19) because we clearly have $4 B^{2}+4 B-1>0$, provided that $B \geq 1$. 
Putting together (2.17), (2.18), and (2.21), we may write $M_{\max }>\exp [-4 B] /(16 B)$, provided that $B>1$. But, requiring $B>1$ is equivalent to $s^{\prime}>128 \sigma^{2} /\left(\beta \Delta^{2} \theta^{2}\right)$. As noted earlier, when $s^{\prime}>8$, we have $\Delta>4$, so it is enough to require that $s^{\prime}>8 \sigma^{2} /\left(\beta \theta^{2}\right)$. Therefore, under the hypotheses of the lemma, $g_{2}\left(s^{\prime}\right)>A\left(s^{\prime}\right) \exp [-4 B] /(16 B)$, from which it follows that

$$
g_{2}\left(s^{\prime}\right)>\frac{512}{9 \pi \Delta^{2}}\left(\frac{x_{0}}{\theta}-1\right)\left(s^{\prime}\right)^{-4} \exp \left[-\frac{\beta}{32}\left(\frac{\theta \Delta}{\sigma}\right)^{2} s^{\prime}\right] .
$$

Since $\Delta\left(s^{\prime}\right)<s^{\prime}$ for $s^{\prime}>0$, and since $\beta>0$, we have

$$
g_{2}\left(s^{\prime}\right)>\frac{512}{9 \pi}\left(\frac{x_{0}}{\theta}-1\right)\left(s^{\prime}\right)^{-6} \exp \left[-\frac{\beta}{32}\left(\frac{\theta}{\sigma}\right)^{2}\left(s^{\prime}\right)^{3}\right],
$$

as required. This completes the proof of Lemma 2.4 .

\section{Strictly positive lower bound for $\rho_{X}$}

The proof of our main theorem follows from Lemma 2.4 and Corollary 2.1.

Proof of Theorem 1.1. Let $\{X(t), t \geq 0\}$ be an OU process satisfying (1.1) and (1.2), and let $\beta, \sigma$, and $x_{0}$ be positive constants; let $\theta$ be a constant satisfying $x_{0}>\theta>0$. To simplify the notation, we introduce the positive constants

$$
K=\frac{512}{9 \pi}\left(\frac{x_{0}}{\theta}-1\right), \quad H=\frac{\beta}{32}\left(\frac{\theta}{\sigma}\right)^{2} .
$$

Let $u=(1 / 2 \beta) \log \left[1+\left\{8 \vee\left(1+x_{0}^{2} / \theta^{2}\right) \vee\left(8 \sigma^{2} /\left(\beta \theta^{2}\right)\right)\right\}\right]$. Then, by virtue of the time rescaling (cf. (2.4)), from Corollary 2.1 and Lemma 2.4, provided that $t>u$, we may write

$$
\begin{aligned}
\rho_{X}(t) & \geq 2 \beta \mathrm{e}^{2 \beta t} g_{2}\left(\mathrm{e}^{2 \beta t}-1\right) \\
& >2 \beta \mathrm{e}^{2 \beta t} K\left(\mathrm{e}^{2 \beta t}-1\right)^{-6} \exp \left[-H\left(\mathrm{e}^{2 \beta t}-1\right)^{3}\right] \\
& >2 \beta K \mathrm{e}^{-10 \beta t} \exp \left[-H \mathrm{e}^{6 \beta t}\right] .
\end{aligned}
$$

Let $p=1+H$ and $k=2 \beta K$. By hypothesis, $s=\left(\mathrm{e}^{2 \beta t}-1\right)>8$, so $\beta t>\log 3>1$. It follows that $\mathrm{e}^{-10 \beta t}>\exp \left[-\mathrm{e}^{6 \beta t}\right]$. Therefore, $\rho_{X}(t)>2 \beta K \exp \left[-(1+H) \mathrm{e}^{6 \beta t}\right]=k \exp \left[-p \mathrm{e}^{6 \beta t}\right]$. This concludes the proof of Theorem 1.1.

The form of the constants and the bound are consistent with the following heuristic relationships between the parameters and the tail of the decay. If $\beta$ is small, the initial condition is far from the threshold, and the noise is large $\left(x_{0}, \sigma \gg \theta\right)$, it is reasonable that the tail of the FPT density should decay relatively slowly. If $\beta$ is large, the initial condition is close to the threshold, and the noise is small, it is reasonable to expect a faster decay of the density at long times.

\section{Discussion}

\subsection{Potential improvements to the construction}

The work reported here does not aim to provide the sharpest possible lower bound to the tail of the FPT density $\rho_{X}$. For reasons discussed further in Section 4.3, our goal was merely to provide some rigorous strictly positive lower bound. It is obvious from inspecting Figure 1 
that the bound may significantly underestimate the FPT density, even for the constants used in the figure. Consider the region bounded by the lines $L_{1}, L_{2}$, and the curve $b(s)$ for $0 \leq s \leq s^{\prime}$. The probability density $g_{2}$ is conditioned so as to eliminate any paths that enter this region, even though many of the paths that make first contact with the curve $b(s)$ in the interval $\left[s^{\prime}, s^{\prime}+\mathrm{d} s\right)$ may pass through it. Clearly, we could get a better approximation by replacing the horizontal line $L_{2}$ with a line intersecting the point $\left(s^{\prime}, b\left(s^{\prime}\right)\right)$ with slope $\mathrm{d} b\left(s^{\prime}\right) / \mathrm{d} s$. The resulting boundary would still yield a convolution of known transition densities, although with a somewhat more complicated form. Going further, we could replace the two component piecewise-linear protective boundary with an $n$-segment piecewise-linear protective boundary. The first segment would have the same slope as $L_{1}$; the last segment would have slope $b^{\prime}\left(s^{\prime}\right)$; intermediate lines could be chosen appropriately.

Indeed, comparison of inequality (1.3) with numerically generated samples from the FPT distribution suggests that the lower bound derived here significantly underestimates the density in the tail of the distribution. Further work in this direction lies beyond the scope of this paper.

\subsection{Relation to other asymptotic FPT density results}

Nobile et al. [14] derived an asymptotic expression for the FPT distribution $\rho_{\theta}\left(t \mid x_{0}\right)$ for an OU process with limiting mean value $x_{\infty}$ to go from initial value $x_{0}$ to a boundary of height $\theta$ in the limit in which $\theta \gg x_{0}, x_{\infty}$. They proved that

$$
\rho_{\theta}\left(t \mid x_{0}\right)=\frac{1}{\tau} \mathrm{e}^{-t / \tau}+o\left(\frac{1}{\tau} \mathrm{e}^{-t / \tau}\right) \quad \text { as } \theta \rightarrow \infty,
$$

where $\tau$ is the mean FPT. These same authors extended this result to a broader class of diffusion processes in [15]. Similarly, Giorno et al. [6] showed that the FPT distribution showed good agreement with the form

$$
\rho(t) \approx Z(t) \mathrm{e}^{-\lambda t}
$$

for passage to a periodically varying boundary $\theta(t)$, in cases where $\theta(t) \gg x_{0}=x_{\infty}$. The limit $\theta \gg x_{0}, x_{\infty}$ corresponds to the subthreshold case, in contrast to the suprathreshold case $x_{0}>\theta>x_{\infty}$ which concerns us here.

Pauwels [17] gave conditions on the noise $(\sigma)$ and drift $(b)$ parameters, guaranteeing that the stochastic differential equation $\left\{\mathrm{d} X_{t}=\sigma\left(X_{t}\right) \mathrm{d} B_{t}+b\left(X_{t}\right) \mathrm{d} t, X_{0}=x_{0}\right\}$ should yield an FPT density, $\rho\left(t, \theta \mid x_{0}\right)$, that is jointly continuous and $k$-fold differentiable in $t, \theta$, and $x_{0}$. Pauwels' conditions are satisfied by the time homogeneous process considered above. In [10] Lehmann used an integral equation approach to extend these results, giving conditions guaranteeing Hölder continuity of the FPT distribution.

Several authors have obtained expressions for moments of the distribution of first passage times, to constant as well as to certain moving barriers, for the time homogeneous OU process. See, for instance, [32] and [33]. Lindner [11] calculated moments of the FPT for both exponentially decaying and periodic driving terms similar to the situation described in (4.1) below. However, knowing the moments of the FPT distribution does not provide a strictly positive lower bound for the tail of the density.

In 1977 Sato proved that the FPT density $\rho_{c}(t)$ for a Wiener process from $B(0)=0$ to a square root boundary $c \sqrt{t+1}$ scales as

$$
\rho_{c}(t) \sim \alpha t^{-p(c)-1},
$$

where $0<p(c)<\frac{1}{2}$, and it is assumed that $c>0$. This provides a lower bound for the tail of the density (in the case considered) since if $\rho_{c}(t) /\left(\alpha t^{-p(c)-1}\right) \rightarrow 1$ as $t \rightarrow \infty$ then, for 
any $\varepsilon>0$, there is a $T$ such that, for $t>T$, we have

$$
\rho_{c}(t)>\frac{\alpha t^{-p(c)-1}}{1+\varepsilon} .
$$

Sato's result does not provide a lower bound for the suprathreshold OU process however. The suprathreshold case corresponds instead to a boundary of the form $-1+c \sqrt{t}$ after appropriate scaling, because in this case the moving boundary crosses the mean value of the Wiener process, and the expected FPT is finite. In the case considered by Sato, the mean FPT is infinite, and the distance from the mean $\mathrm{E}[B]=0$ to the boundary grows monotonically.

\subsection{Prospective application to neural synchronization}

Strictly positive lower bounds are also of interest in the case of OU processes with time varying forcing. The timing of action potentials in nerve cells stimulated by fluctuating current injections are of significant interest in neuroscience [7], [13], [19], [29]. The timing of individual spikes in relation to ongoing endogenous rhythmic activity is of particular interest [24], [30], as is the reliable occurrence of specific patterns involving multiple spikes in succession [5], [31]. There are many models available for theoretical studies of the synchronization of nerve cells, but, for models incorporating the effects of noise, the LIF model remains among the most tractable, attracting sustained attention [23], [26], [27]. For this case, the model dynamics in (1.1) are extended to incorporate the drive through the time varying function $h(t)$, assumed to be bounded, measurable, and of zero mean:

$$
\mathrm{d} X=(-\beta X+h(t)) \mathrm{d} t+\sigma \mathrm{d} W
$$

When the forcing function $h$ is periodic with period $T$, we are interested in the succession of FPTs $\left\{\tau_{1}, \tau_{2}, \ldots\right\}$, assuming that the process is instantaneously reset to $x_{0}$ upon each encounter with the threshold. It can be shown that the sequence of phases of the boundary crossings relative to the periodic drive, $\phi_{n}=\tau_{n} \bmod T$, then forms a Markov process on the circle $\mathbb{S}^{1} \equiv[0,1)$, with transition probabilities given by a map $\mathbb{K}: f_{k} \rightarrow f_{k+1}$, where

$$
f_{k+1}(\phi)=\int_{\psi \in \mathbb{S}^{1}} K(\phi, \psi) f_{k}(\psi) \mathrm{d} \psi .
$$

Here $f_{k}(\phi)$ is the density function for the phase of the $k$ th boundary crossing relative to the periodic drive. This framework has been elaborated to study stochastic synchronization of systems analogous to the LIF model neuron driven by periodic injected current [28] as well as LIF model neurons with constant drive and periodically varying $\theta(t)$ [23], [25], [26]. In both types of model, numerical investigations suggest that, regardless of initial conditions, the phase distribution converges to a unique probability density on the circle [23], [28]. This situation contrasts with that of the deterministic periodically forced LIF model neuron, in which a rich collection of resonances and $p: q$ mode locking are known to occur [2], [8], [16], [20]. The existence of $p: q$ mode locked solutions ( $q$ boundary crossings per $p$ stimulus periods) implies the existence of multiple invariant measures on the circle when $p>1$.

Tateno et al. [28] conjectured that the operator $\mathbb{K}$ for the stochastic periodically forced LIF model neuron has a unique invariant density and that the sequence of probability densities $\left\{\mathbb{K}^{n} f_{0}\right\}$ is asymptotically stable (see [28, Sections 5-6]). One approach to proving this conjecture would be to exploit the following result (with $m=1$ ). 
Theorem 4.1. (Corollary 5.7.1 of [9]; see also [28, Equation (38)].) Let (X, A, $\mu$ ) be a measure space, let $K: X \times X \rightarrow \mathbb{R}$ be a stochastic kernel, and let $\mathbb{K}$ be the corresponding Markov operator (defined by the integral equation (4.2)). Denote by $K_{n}$ the kernel corresponding to $\mathbb{K}^{n}$. If, for some $m$,

$$
\int_{X} \inf _{y} K_{m}(x, y) \mathrm{d} x>0
$$

then $\left\{P^{n}\right\}$ is asympotically stable.

Exhibiting a strictly positive lower bound analogous to (1.3) for the periodically forced case would immediately establish the infimum criterion (4.3). The criterion has been assumed to hold under various circumstances (cf. [4, Equation (6)], [23, p. 330], and [25, Equation (29)]), but, to the author's knowledge, an explicit lower bound for the FPT density for a suprathreshold system has not been obtained either for the periodically forced LIF model with constant boundary or for the time homogeneous LIF model with oscillating boundary. The extension of Theorem 1.1 to one or another of these cases remains a topic for future research.

\section{Acknowledgements}

This work was supported in part by NSF grants DMS-0720142 and DMS-1010434 in the mathematical biology program. I am grateful for discussion and encouragement to the following: D. Calvetti, M. Denker, R. Galan, K. Kirkpatrick, P. Kotelenez, K. Loparo, E. Somersalo, S. Szarek, and W. Woyczynski. I thank an anonymous referee for providing valuable feedback. I am grateful to the Oberlin College Library for research support.

\section{References}

[1] Capocelli, R. M. and Ricciardi, L. M. (1971). Diffusion approximation and first passage time problem for a model neuron. Kybernetik 8, 214-223.

[2] Coombes, S. And Bressloff, P. C. (1999). Mode locking and Arnold tongues in integrate-and-fire neural oscillators. Phys. Rev. E 60, 2086-2096.

[3] Cox, D. R. And Miller, H. D. (1965). The Theory of Stochastic Processes. John Wiley, New York.

[4] Doi, S., Inoue, J. and Kumagai, S. (1998). Spectral analysis of stochastic phase lockings and stochastic bifurcations in the sinusoidally forced van der Pol oscillator with additive noise. J. Statist. Phys. 90, 1107-1127.

[5] Fellous, J.-M., Tiesinga, P. H. E., Thomas, P. J. And Sejnowski, T. J. (2004). Discovering spike patterns in neuronal responses. J. Neurosci. 24, 2989-3001.

[6] Giorno, V., Nobile, A. G. and Ricciardi, L. M. (1990). On the asymptotic behaviour of first-passage-time densities for one-dimensional diffusion processes and varying boundaries. Adv. Appl. Prob. 22, 883-914.

[7] Hunter, J. D., Milton, J. G., Thomas, P. J. and Cowan, J. D. (1998). Resonance effect for neural spike time reliability. J. Neurophysiology 80, 1427-1438.

[8] Keener, J. P., Hoppensteadt, F. C. and Rinzel, J. (1981). Integrate-and-fire models of nerve membrane response to oscillatory input. SIAM J. Appl. Math. 41, 503-517.

[9] Lasota, A. And Mackey, M. C. (1994). Chaos, Fractals, and Noise (Appl. Math. Sci. 97). Springer, New York.

[10] Lehmann, A. (2002). Smoothness of first passage time distributions and a new integral equation for the first passage time density of continuous Markov processes. Adv. Appl. Prob. 34, 869-887.

[11] Lindner, B. (2004). Moments of the first passage time under external driving. J. Statist. Phys. 117, $703-737$.

[12] LoAder, C. R. AND DeELY, J. J. (1987). Computations of boundary crossing probabilities for the Wiener process. J. Statist. Comput. Simul. 27, 95-105.

[13] Mainen, Z. F. and Sejnowski, T. J. (1995). Reliability of spike timing in neocortical neurons. Science 268, 1503-1506.

[14] Nobile, A. G., Ricciardi, L. M. and Sacerdote, L. (1985). Exponential trends of Ornstein-Uhlenbeck firstpassage-time densities. J. Appl. Prob. 22, 360-369.

[15] Nobile, A. G., Ricciardi, L. M. and Sacerdote, L. (1985). Exponential trends of first-passage-time densities for a class of diffusion processes with steady-state distribution. J. Appl. Prob. 22, 611-618.

[16] Pakdaman, K. (2001). Periodically forced leaky integrate-and-fire model. Phys. Rev. E 63, 041907, 5pp.

[17] Pauwels, E. J. (1987). Smooth first-passage densities for one-dimensional diffusions. J. Appl. Prob. $24,370-377$. 
[18] Pinsky, M. And Karlin, S. (2011). An Introduction to Stochastic Modeling, 4th edn. Academic Press, Burlington, CA.

[19] Reinagel, P. AND ReID, R. C. (2002). Precise firing events are conserved across neurons. J. Neurosci. 22, 6837-6841.

[20] Rescigno, A., Stein, R. B., Purple, R. L. And Poppele, R. E. (1970). A neuronal model for the discharge patterns produced by cyclic inputs. Bull. Math. Biophys. 32, 337-353.

[21] Ricciardi, L. M. and Sato, S. (1988). First-passage-time density and moments of the Ornstein-Uhlenbeck process. J. Appl. Prob. 25, 43-57.

[22] SATo, S. (1977). Evaluation of the first-passage time probability to a square root boundary for the Wiener process. J. Appl. Prob. 14, 850-856.

[23] Shimokawa, T. et al. (2000). A first-passage-time analysis of the periodically forced noisy leaky integrate-andfire model. Biol. Cybernet. 83, 327-340.

[24] Stiefel, K. M., Fellous, J. M., Thomas, P. J. And Sejnowski, T. J. (2010). Intrinsic subthreshold oscillations extend the influence of inhibitory synaptic inputs on cortical pyramidal neurons. Eur. J. Neurosci. 31, 1019-1026.

[25] Tateno, T. (1998). Characterization of stochastic bifurcations in a simple biological oscillator. J. Statist. Phys. 92, 675-705.

[26] Tateno, T. (2002). Noise-induced effects on period-doubling bifurcation for integrate-and-fire oscillators. Phys. Rev. E 65, 021901, 10pp.

[27] Tateno, T. and Jimbo, Y. (2000). Stochastic mode-locking for a noisy integrate-and-fire oscillator. Phys. Lett. A 271, 227-236.

[28] Tateno, T., Doi, S., Sato, S. and Ricciardi, L. M. (1995). Stochastic phase lockings in a relaxation oscillator forced by a periodic input with additive noise: a first-passage-time approach. J. Statist. Phys. 78, 917-935.

[29] Thomas, P. J., Tiesinga, P. H., Fellous, J. M. and Sejnowski, T. J. (2003). Reliability and bifurcation in neurons driven by multiple sinusoids. Neurocomput. 52-54, 955-961.

[30] Tiesinga, P., Fellous, J.-M. And Sejnowski, T. J. (2008). Regulation of spike timing in visual cortical circuits. Nature Rev. Neurosci. 9, 97-107.

[31] Toups, J. V. et al. (2011). Finding the event structure of neuronal spike trains. To appear in Neural Computation.

[32] Tuckwell, H. C. and Wan, F. Y. M. (1984). First-passage time of Markov processes to moving barriers. J. Appl. Prob. 21, 695-709.

[33] Wan, F. Y. M. and Tuckwell, H. C. (1982). Neuronal firing and input variability. J. Theoret. Neurobiol. 1, 197-218. 